\title{
Modelos matemáticos para predição da chuva de projeto para regiões do Estado de Minas Gerais
}

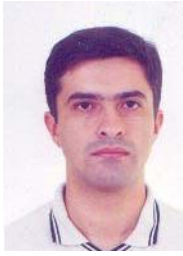

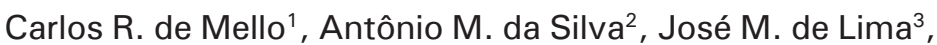
Daniel F. Ferreira ${ }^{4} \&$ Marcelo S. de Oliveira ${ }^{4}$

\author{
1 DCS/UFLA. Campus Universitário, CP 37, CEP 37200-000, Lavras, MG. Fone: (35) 3821-7143. E-mail: crmello@ufla.br (Foto) \\ 2 DEG/UFLA. Fone: (35) 3829-1386. E-mail: marciano@ufla.br \\ ${ }^{3}$ DCS/UFLA. Fone: (35) 3829-1332. E-mail: jmlima@ufla.br \\ ${ }^{4}$ DEX/UFLA. Fones: (35) 3829-1369 ou 3829-1373. E-mail: danielff@ufla.br, marcelso@ufla.br
}

Protocolo $111-6 / 8 / 2002$ - Aprovado em 7/2/2003

\begin{abstract}
Resumo: O uso de modelos matemáticos para predição da chuva é uma forma prática e precisa para determinação do valor a ser aplicado em projetos, sendo útil para localidades desprovidas de informações pluviométricas. Objetivou-se ajustar o método de Bell, que possui características de regionalização para a chuva de projeto, com base em equações de chuvas intensas e modelos de probabilidade de Gumbel de estações meteorológicas do Estado de Minas Gerais ajustando, também, um modelo para cada região do estado. Avaliaram-se os modelos considerando-se o coeficiente de determinação e os erros médios em relação aos dados originais. Para validação, trabalhou-se com três estações meteorológicas da região Norte não usadas para ajuste do respectivo modelo. Foram analisadas três metodologias para estimativa da chuva intensa padrão $\left(\mathrm{h}_{(60,2)}\right)$, que pondera o método usado, ressaltando-se a média aritmética, a média ponderada pelo inverso do quadrado da distância e a predição geoestatística (krigagem). Observou-se que os modelos possuem bons indicadores estatísticos e a validação produziu erros baixos, mostrando que os modelos podem ser aplicados, especialmente se a krigagem for usada para estimativa do parâmetro $h_{(60,2)}$.
\end{abstract}

Palavras-chave: precipitação intensa, obras hidráulicas, regionalização, krigagem

\section{Mathematical models for the estimation of rainfall in selected regions of Minas Gerais State, Brazil}

\begin{abstract}
The use of mathematical models for predicting rainfall is a practical and accurate way of determining this parameter to be applied to regions which do not have any precipitation data. Based on the intense rainfall equations and Gumbel's probability model for maximum daily precipitation of meteorological stations in Minas Gerais State, the objective of this work was to adjust the Bell's Method, with regional features, for rainfall, adjusting one model for each region. The regional parameters were estimated by non-linear multiple regression, using Gauss-Newton's method. The goodness of the models was evaluated by the coefficient of determination and mean errors of prediction as compared to the original data. Data from three meteorological stations in the Northern region, which were not used to adjust the respective model, were used for validation purposes. The most frequent precipitation was tested by the arithmetic mean, the weighted mean by the inverse-square-distance and the geo-statistical prediction (kriging). The models produced good statistical parameters, with low mean errors, showing their accuracy, specially when the kriging method for estimating the most frequent precipitation was used.
\end{abstract}

Key words: intense precipitation, hydraulic structures, regionalization, kriging

\section{INTRODUÇÃO}

A determinação de boas estimativas de variáveis físicas é o ponto base e mais importante para o desenvolvimento de projetos na engenharia. Estruturas hidráulicas destinadas à contenção do excesso de chuva, como barragens, bacias de contenção, terraços e outras, são projetadas mediante o uso de um bom estimador para uma precipitação que satisfaça a segurança desejada e promova um projeto economicamente racional.

No meio rural, normalmente as obras hidráulicas estão inseridas em bacias hidrográficas de pequeno porte e pouco habitadas, sendo que as precipitações são consideradas com duração inferior a 120 min, e a probabilidade de ocorrência do evento, expressa pelo tempo de retorno, geralmente inferior a 20 anos (Chow et al., 1988; Pinto et al., 1996; Brooks et al., 1997). 
O uso de equações matemáticas que relacionem a chuva de projeto às variáveis tempo de retorno e tempo de duração, tem sido o mecanismo mais prático usado em projetos hidroagrícolas. Um dos problemas é que a disponibilidade de dados de chuva é bastante restrita no Brasil sendo, na maioria das vezes, inexistente para determinada região ou de acesso restrito, em especial para particulares (Mello et al., 2001).

A equação de chuvas intensas é uma metodologia bastante utilizada que considera as variáveis destacadas anteriormente, porém para ajuste do modelo, são necessárias séries históricas de dados de precipitação máxima diária anual de cada localidade específica, além de ser válida apenas para o local em que foi ajustada, haja vista que este modelo não apresenta características de regionalização, sendo detectadas altas variabilidades dos parâmetros entre as estações (Denardin \& Freitas, 1982; Yue, 2001; Heneker et al., 2001).

O uso da espacialização dos parâmetros de equações de chuvas intensas, conforme proposto por Silva et al. (1999) também é uma alternativa quando não se dispõe de dados. No entanto, deve-se considerar que tal espacialização vem sendo feita com base no inverso do quadrado da distância euclidiana e essa metodologia pode apresentar imprecisões consideráveis, por não ser o melhor método estatístico para esse fim, haja vista que a krigagem geoestatística pode promover melhorias consideráveis na precisão, por ser baseada na condição de variância mínima e não tendenciosidade, reduzindo erros aleatórios através do controle de uma parcela desse erro, representada pela influência da posição das amostras (Assad et al., 1994; Gotway et al., 1996; Vieira, 2000).

Bell (1969) propôs uma metodologia que tem a vantagem de ser ajustada para regiões e pondera o valor da chuva de projeto por uma precipitação intensa padrão, cujo tempo de retorno é de 2 anos e tempo de duração $60 \mathrm{~min}$, fazendo com que o valor predito possa ser mais coerente, tanto do ponto de vista físico quanto do econômico, para o projeto. $\mathrm{O}$ tempo de duração, 60 min, é uma referência de duração para as chuvas em que, de acordo com trabalhos relatados por Froehlich (1993), Nobukuni (2000) e Cruciani et al. (2002) a freqüência de ocorrência para chuvas com essa duração é bastante elevada. Esta metodologia foi aplicada e testada por Pegoraro (1996) para a região metropolitana de Campinas, obtendo-se bons resultados de predição, o que encoraja sua aplicação, tendo em vista as vantagens práticas produzidas por uma equação de chuvas intensas generalizada para uma região e com precisão.

Assim, observa-se que um modelo em que se possa relacionar um valor de chuva de projeto às variáveis tempo de retorno e tempo de duração, ponderada por uma precipitação de $60 \mathrm{~min}$, e, ainda, que pode ser ajustado para regiões, constitui-se de uma ferramenta preditora que pode ser poderosa no auxílio a projetos hidroagrícolas, sendo aplicável a localidades que não dispõem de informações hidrológicas, desde que a precipitação $\mathrm{h}_{60,2}$ tenha boa estimativa.

Os objetivos deste trabalho foram: ajustar o modelo proposto por Bell (1969) para predição da chuva de projeto, para as regiões fisiográficas do Estado de Minas Gerais (Sul, Leste, Norte, Centro e Triângulo Mineiro) proporcionando, desta forma, predições confiáveis para localidades onde não há registros históricos de precipitação, e comparar metodologias distintas para estimativa do parâmetro $\mathrm{h}_{(60,2)}$, sendo de suma importância a sua determinação de forma mais precisa possível.

\section{MATERIAL E MÉTODOS}

\section{Descrição do método de Bell (1969)}

Righetto (1998) cita algumas características desta metodologia, entre elas o fato da equação exigir a determinação de apenas uma variável física, $\mathrm{o}_{(60,2)}$. A Eq. 1 representa a forma geral proposta:

$$
\mathrm{h}_{(\mathrm{td}, \mathrm{TR})}=\left(\mathrm{a} \ln \mathrm{TR}+\mathrm{a}_{1}\right)\left(\mathrm{a}_{2} \cdot \mathrm{td}^{\mathrm{b}}-\mathrm{a}_{3}\right) \mathrm{h}_{(60,2)}
$$

em que:

$\mathrm{h}_{(\mathrm{td}, \mathrm{TR})}$ - chuva ou precipitação de projeto, $\mathrm{mm}$

TR - tempo de retorno, anos

Td - tempo de duração, min

$\mathrm{a}, \mathrm{a}_{1}, \mathrm{a}_{2}, \mathrm{a}_{3}$ e b - parâmetros regionais de ajuste do modelo

$\mathrm{h}_{(60,2)}$ - precipitação intensa padrão que corresponde à duração de 60 min e tempo de retorno de 2 anos, $\mathrm{mm}$

Ainda de acordo com Righetto (1998) esta equação foi ajustada com base em dados de postos brasileiros, chegandose à seguinte forma geral, para o Brasil:

$$
\mathrm{h}_{(\mathrm{td}, \mathrm{TR})}=(0,31 \ln \mathrm{TR}+0,7)\left(0,38 \cdot \mathrm{td}^{0,31}-0,39\right) \mathrm{h}_{(60,2)}
$$

$\mathrm{O}$ valor de $\mathrm{h}_{(60,2)}$ pode ser determinado da seguinte forma:

$$
\mathrm{h}_{(60,2)} \cong \mathrm{Kh}_{(\mathrm{dia}, 2)}
$$

donde:

$$
\begin{aligned}
& \mathrm{K} \quad-0,510 \\
& \mathrm{~h}_{(\mathrm{dia}, 2)} \text { - altura pluviométrica máxima diária anual correspon- } \\
& \text { dente ao tempo de retorno de } 2 \text { anos, } \mathrm{mm}
\end{aligned}
$$

Ressalta-se que, segundo Bertoni \& Tucci (2001) este modelo só é válido para chuvas de duração inferior a 120 min e tempo de retorno entre 2 e 20 anos.

\section{Separação das regiões do Estado de Minas Gerais}

O Estado de Minas Gerais foi dividido em 5 regiões: Sul, Norte, Leste, Triângulo Mineiro e Centro adotando-se, como critério, as posições geográficas e características fisiográficas típicas, segundo abordagem dada por Moreira (1997), como relevo, cobertura vegetal e características pluviométricas, esta última característica baseada em trabalho de Almeida (1995) e em consulta ao $5^{\circ}$ Distrito de Meteorologia, em Belo Horizonte. Tal abordagem permite associar-se um caráter climático mais homogêneo para as regiões, no tocante à gênese das chuvas intensas. As principais características fisiográficas das regiões e a classificação climática, segundo Köppen, podem ser resumidas da seguinte forma: 
Região Norte: caracterizada por relevo com pequena presença de grandes cadeias montanhosas, com altitudes próximas entre as estações, clima tendendo a semi-árido (déficit hídrico superior a 3 meses do ano) e cobertura vegetal típica de cerrado e caatinga. A precipitação total anual varia de 900 a $1100 \mathrm{~mm}, \mathrm{o}$ número total de dias chuvosos de 60 a 80 e tipo climático Aw. Geograficamente, localizada em latitudes inferiores a $18^{\circ} \mathrm{S}$.

Região Central: evidenciada por um relevo movimentado, com altitudes acima de $500 \mathrm{~m}$ e cobertura vegetal tendendo a cerrado. Precipitação total anual de 1100 a $1500 \mathrm{~mm}$, número total de dias chuvosos de 81 a 100 dias e classificação climática, Cwa com transição para Aw, localizando-se aproximadamente entre as coordenadas geográficas 18 e $21^{\circ} \mathrm{S}$ e 43 e $46^{\circ} \mathrm{W}$.

Região Triângulo Mineiro: caracterizada por relevo típico de chapada, conferindo altitudes elevadas, com cobertura vegetal tipicamente de cerrado, período de déficit hídrico marcante nos meses de inverno. Precipitação total anual de 1300 a $1500 \mathrm{~mm}$, número de dias chuvosos de 91 a mais de 100 dias e classificação climática entre Cwa e Aw, localizando-se entre as coordenadas geográficas 18 e $20^{\circ} \mathrm{S}$ e 46 e $50^{\circ} \mathrm{W}$.

Região Sul: assinalada por relevo montanhoso com influência de altas cadeias montanhosas, conferindo altitudes médias elevadas, raramente inferiores a $800 \mathrm{~m}$, além de cobertura vegetal oscilando entre remanescentes de mata atlântica e o cerrado. Possui as menores temperaturas médias do Estado, com formação freqüente de geadas nos meses de maio a julho e déficit hídrico de julho a setembro. Precipitação total anual próxima de $1500 \mathrm{~mm}$, total de dias chuvosos acima de 100 dias e classificação climática $\mathrm{Cwb}$, localizando-se a latitudes superiores a $21^{\circ} \mathrm{S}$ e longitudes entre 43 e $46^{\circ} \mathrm{W}$.

Região Leste: caracterizada por relevo movimentado e altitudes inferiores a $700 \mathrm{~m}$, conhecido como Mar de Morros, com cobertura vegetal próxima à mata atlântica. Precipitação total anual de 1100 a $1500 \mathrm{~mm}$, total de dias chuvosos entre 70 e 100 dias e classificação climática entre Cwa e Aw, localizada entre as coordenadas geográficas 18 e $21^{\circ} \mathrm{S}$ e 41 e $43^{\circ} \mathrm{W}$.

De maneira geral, o Estado de Minas Gerais apresenta, em todas as suas regiões, chuvas irregularmente distribuídas, com concentração de $90 \%$ do total precipitado e das chuvas intensas nos meses de outubro a março.

$\mathrm{Na}$ Tabela 1 e Figura 1, observaram-se as cidades (estações meteorológicas) que representaram cada região, suas respectivas coordenadas geográficas e localização dentro do Estado.

\section{Obtenção dos dados e ajuste dos modelos}

Através de equações de chuvas intensas ajustadas para cada estação meteorológica, desenvolvidas por Pinto (1995) trabalhou-se com tempo de retorno variando de 2 a 20 anos e tempo de duração de 5 a $120 \mathrm{~min}$, obtendo-se as chuvas máximas e a chuva intensa padrão $\left(\mathrm{h}_{(60,2)}\right)$ para cada localidade da Tabela 1. Através de modelos de distribuição de probabilidades de Gumbel ajustados e testadas suas adequacidades pelo citado autor, para chuvas máximas diárias associadas aos
Tabela 1. Estações meteorológicas utilizadas, respectivas regiões e coordenadas geográficas

\begin{tabular}{|c|c|c|c|}
\hline Local $^{*}$ & Latitude & Longitude & Altitude (m) \\
\hline \multicolumn{4}{|l|}{ Região Norte } \\
\hline 7 - Teófilo Otoni & $17^{\circ} 51^{\prime} \mathrm{S}$ & $41^{\circ} 31^{\prime} \mathrm{W}$ & 356,38 \\
\hline 5 - Pirapora & $17^{\circ} 20^{\prime} \mathrm{S}$ & $44^{\circ} 57^{\prime} \mathrm{W}$ & 505,24 \\
\hline 8 - Salinas & $16^{\circ} 10^{\prime} \mathrm{S}$ & $42^{\circ} 18^{\prime} \mathrm{W}$ & 471,32 \\
\hline 9 - Januária & $15^{\circ} 26^{\prime} \mathrm{S}$ & $44^{\circ} 22^{\prime} \mathrm{W}$ & 473,71 \\
\hline 10 - Montes Claros & $16^{\circ} 43^{\prime} \mathrm{S}$ & $43^{\circ} 52^{\prime} \mathrm{W}$ & 646,29 \\
\hline \multicolumn{4}{|l|}{ Região Sul } \\
\hline 1 - Lavras & $21^{\circ} 14^{\prime} \mathrm{S}$ & $45^{\circ} 00^{\prime} \mathrm{W}$ & 918,84 \\
\hline 2 - Machado & $21^{\circ} 40^{\prime} \mathrm{S}$ & $45^{\circ} 55^{\prime} \mathrm{W}$ & 873,35 \\
\hline 3 - Caxambu & $21^{\circ} 58^{\prime} \mathrm{S}$ & $44^{\circ} 56^{\prime} \mathrm{W}$ & 958,51 \\
\hline 16 - Barbacena & $21^{\circ} 15^{\prime} \mathrm{S}$ & $45^{\circ} 59^{\prime} \mathrm{W}$ & 1126,87 \\
\hline \multicolumn{4}{|l|}{ Região do Triângulo Mineiro } \\
\hline 11 - Capinópolis & $18^{\circ} 41^{\prime} \mathrm{S}$ & $49^{\circ} 34^{\prime} \mathrm{W}$ & 620,60 \\
\hline 12 - Uberlândia & $18^{\circ} 55^{\prime} \mathrm{S}$ & $48^{\circ} 17^{\prime} \mathrm{W}$ & 872,00 \\
\hline 13 - Uberaba & $19^{\circ} 45^{\prime} \mathrm{S}$ & $47^{\circ} 55^{\prime} \mathrm{W}$ & 742,90 \\
\hline 14 - Araxá & $19^{\circ} 34^{\prime} \mathrm{S}$ & $46^{\circ} 56^{\prime} \mathrm{W}$ & 1003,28 \\
\hline 15 -Patos de Minas & $18^{\circ} 36^{\prime} \mathrm{S}$ & $46^{\circ} 31^{\prime} \mathrm{W}$ & 940,28 \\
\hline \multicolumn{4}{|l|}{ Região Leste } \\
\hline 22 - Governador Valadares & $18^{\circ} 51^{\prime} \mathrm{S}$ & $41^{\circ} 56^{\prime} \mathrm{W}$ & 277,45 \\
\hline 6 - Viçosa & $20^{\circ} 45^{\prime} \mathrm{S}$ & $42^{\circ} 51^{\prime} \mathrm{W}$ & 689,73 \\
\hline 17 - Caratinga & $19^{\circ} 48^{\prime} \mathrm{S}$ & $45^{\circ} 09^{\prime} \mathrm{W}$ & 609,65 \\
\hline 18 - Aimorés & $19^{\circ} 29^{\prime} \mathrm{S}$ & $41^{\circ} 04^{\prime} \mathrm{W}$ & 82,74 \\
\hline \multicolumn{4}{|l|}{ Região Centro } \\
\hline 4 - Diamantina & $18^{\circ} 15^{\prime} \mathrm{S}$ & $43^{\circ} 36^{\prime} \mathrm{W}$ & 1296,12 \\
\hline 20 - Sete Lagoas & $19^{\circ} 28^{\prime} \mathrm{S}$ & $44^{\circ} 15^{\prime} \mathrm{W}$ & 732,00 \\
\hline 21 - Belo Horizonte & $19^{\circ} 56^{\prime} \mathrm{S}$ & $43^{\circ} 56^{\prime} \mathrm{W}$ & 915,00 \\
\hline 19 - Bambuí & $20^{\circ} 00^{\prime} \mathrm{S}$ & $45^{\circ} 59^{\prime} \mathrm{W}$ & 661,27 \\
\hline
\end{tabular}

* O número refere-se a localização na Figura

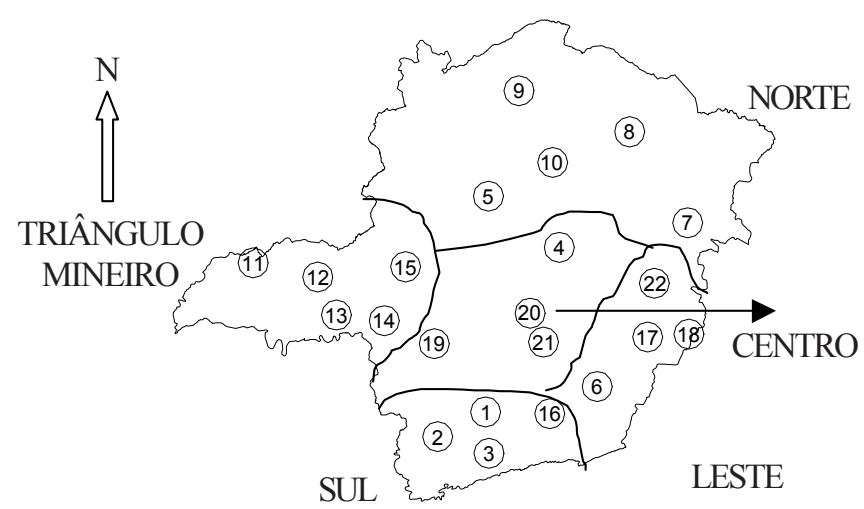

Obs.: Para o nome do local consultar Tabela 1

Figura 1. Mapa do Estado de Minas Gerais e as estações meteorológicas utilizadas em cada região (sem escala)

níveis de probabilidade destacados acima (2 a 20 anos), foi possível determinar-se o parâmetro $h_{(\mathrm{dia}, 2)}$ da Eq. 3. Desta forma, o fator $\mathrm{K}$ foi calculado para cada localidade.

Dispondo-se dos dados de chuvas intensas, período de retorno, tempo de duração e $\mathrm{h}_{(60,2)}$, estimaram-se os parâmetros regionais da Eq. 1, por meio de regressão múltipla não-linear, pelo método de Gauss-Newton (Gallant, 1987) utilizando-se o programa SAS (1985) e se ajustando um modelo específico para cada região do Estado de Minas Gerais.

Os parâmetros estatísticos estimados e considerados na análise da precisão dos modelos foram o coeficiente de determinação ajustado $\left(\mathrm{R}^{2}\right)$ e o erro médio produzido pelos modelos em relação aos valores originais. Além disso, avaliou- 
se a predição proporcionada pelo modelo geral proposto (Eq. 2 ), fazendo-se uma abordagem dos erros proporcionados.

\section{Métodos estatísticos para estimativa do parâmetro $\mathbf{h}_{(60,2)}$.}

Foi desenvolvido um estudo de metodologias para estimativa do parâmetro $\mathrm{h}_{(60,2)}$, analisando-o através de média aritmética dos valores de cada estação utilizada no ajuste do modelo, através de média ponderada pelo inverso do quadrado da distância euclidiana, entre a estação a ser aplicada e as estações meteorológicas utilizadas no ajuste, e por meio de krigagem geoestatística, após estudo da dependência espacial deste parâmetro, para o estado de Minas Gerais (Haan, 1979; Vieira et al., 1991; Gotway et al., 1996; Silva et al., 1999).

A krigagem geoestatística consiste do preditor geoestatístico, o qual estima uma variável num determinado local, atribuindo pesos para cada um dos pontos vizinhos a este local. Esses pontos constituem a vizinhança de krigagem. Isto é possível somente após detectar se há influência da posição nos valores da variável em estudo, o qual é obtido somente após estudo da estrutura de dependência espacial dos dados (Vieira, 2000).

Conforme Thompson (1992) a predição de um ponto por meio de geoestatística, partindo-se de um semivariograma, pode ser feita da seguinte forma:

$$
\begin{aligned}
& {[\mathrm{A}]^{-1}[\mathrm{~B}]=[\lambda]} \\
& \mathrm{Xp}=[\mathrm{X}][\lambda]
\end{aligned}
$$

em que:

$[A]^{-1}$ - representa a matriz inversa dos dados de semivariância, obtidos a partir da distância euclidiana entre cada cidade utilizada na vizinhança de krigagem, aplicada ao modelo de semivariograma

[B] - representa a matriz das semivariâncias obtidas também através do modelo de semivariograma entre cada localidade da vizinhança de krigagem e a localidade a ser estimada

[ $\lambda]$ - representa a matriz dos pesos de krigagem, que são determinados para cada cidade utilizada na vizinhança de krigagem

Xp - variável a ser predita

[X] - matriz dos valores da variável para cada localidade da vizinhança de krigagem.

Para predição geoestatística do parâmetro $\mathrm{h}_{(60,2)}$ foram utilizadas as estações cujas distâncias são inferiores ao alcance encontrado pelo modelo de semivariograma, haja vista que a dependência espacial só é verificada dentro do raio correspondente ao alcance.

Para validação e análise dos resultados obtidos pelas três metodologias de estimação da chuva intensa padrão, fez-se um estudo dos erros gerados pelo modelo ajustado para a região Norte em relação aos dados de precipitação máxima de três estações meteorológicas desta região, que não foram utilizadas para obtenção das estimativas dos parâmetros regionais.

\section{RESULTADOS E DISCUSSÃO}

Na Tabela 2 apresenta-se o modelo ajustado para cada região do Estado de Minas Gerais e as estimativas estatísticas dos indicadores da qualidade do ajuste para cada um deles.

Tabela 2. Modelos ajustados para cada região e indicadores

\begin{tabular}{|c|c|c|c|c|}
\hline Região & Modelo & $\mathrm{R}^{2}$ & E1 & E2 \\
\hline Norte & $\begin{array}{l}\mathrm{h}(\mathrm{td}, \mathrm{TR})=(0,898 \ln \mathrm{TR}+2,134) . \\
\left(0,38 \mathrm{td}^{0,178}-0,439\right) \cdot \mathrm{h}_{(60,2)}\end{array}$ & 0,990 & 5,6 & 7,6 \\
\hline Sul & $\begin{array}{l}\mathrm{h}(\mathrm{td}, \mathrm{TR})=(1,750 \ln \mathrm{TR}+3,821) \\
\left(0,38 \mathrm{td}^{0,116}-0,422\right) \cdot \mathrm{h}_{(60,2)}\end{array}$ & 0,985 & 7,6 & 10,0 \\
\hline Centro & $\begin{array}{l}\left.\mathrm{h}_{(\mathrm{td}, \mathrm{TR}}\right)=(0,719 \ln \mathrm{TR}+1,500) . \\
\left(0,38 \mathrm{td}{ }^{0,219}-0,451\right) \cdot \mathrm{h}_{(60,2)}\end{array}$ & 0,982 & 7,3 & 11,2 \\
\hline Leste & $\begin{array}{l}\mathrm{h}(\mathrm{td}, \mathrm{TR})=(2,088 \ln \mathrm{TR}+4,609) . \\
(0,38 \mathrm{td} 0,098-0,409) \cdot \mathrm{h}_{(60,2)}\end{array}$ & 0,984 & 6,5 & 10,4 \\
\hline $\begin{array}{l}\text { Triângulo } \\
\text { Mineiro }\end{array}$ & $\begin{array}{l}\mathrm{h}(\mathrm{td}, \mathrm{TR})=(0,699 \ln \mathrm{TR}+1,873) . \\
\left(0,38 \mathrm{td} \mathrm{d}^{0,198}-0,445\right) \cdot \mathrm{h}_{(60,2)}\end{array}$ & 0,980 & 7,5 & 10,8 \\
\hline
\end{tabular}
estatísticos* de qualidade proporcionados

${ }^{*} \mathrm{R}^{2}$ - coeficiente de determinação ajustado

E1 - erro médio proporcionado pelo respectivo modelo ajustado em relação aos valores originais (\%) E2 - erro médio proporcionado pelo modelo proposto por Bell (Eq. 2) em relação aos valores originais $(\%)$

Observa-se que os ajustes dos modelos proporcionaram coeficientes de determinação elevados e altamente significativos, refletindo a boa qualidade do ajuste; além disso, os erros médios proporcionados são baixos. Verificou-se, ainda, que o modelo geral (Eq. 2) proporcionou erros médios superiores para todas as regiões, sendo a menor diferença constatada para a região Norte. Avalia-se, também, que o melhor ajuste foi obtido para a região Norte, o que pode estar associado à menor variabilidade do parâmetro $\mathrm{h}_{(60,2)}$, conforme Tabela 3 . Desta forma, as estimativas dos parâmetros estatísticos mostram correlação muito forte entre as variáveis $\mathrm{TR}, \mathrm{td} \mathrm{e} \mathrm{h}_{(60,2)}$ e a variável chuva de projeto, permitindo analisar-se que os modelos ajustados proporcionam predições que são mais precisas e específicas para cada região.

$\mathrm{Na}$ Tabela 3 é possível observar-se os valores médios e o coeficiente de variação para o fator $\mathrm{K}, \mathrm{h}_{(\mathrm{dia}, 2)}$ e $\mathrm{h}_{(60,2)}$ para cada região.

Constata-se que o fator K médio foi, em todas as situações, superior ao valor 0,51 proposto para o Brasil como um todo, $\mathrm{e}$ também uma variabilidade que, em termos hidrológicos, é pequena dentro de cada região (Haan, 1979; Yevjevich, 1982) com coeficiente de variação máximo de $13,4 \%$ para a região Sul. Avaliando-se o $h_{(60,2)}$ e o $h_{(\text {dia, } 2)}$ observa-se, de maneira semelhante, pequena variabilidade dos valores dentro de cada região e, desta forma, analisa-se que as regiões em que o Estado foi dividido com base em características fisiográficas, pluviométricas e tipos climáticos, apresentam boa homogeneidade em termos de precipitação intensa, cuja variação máxima detectada foi de $16 \%$ para a região Sul, o que é considerado baixo quando se monitora variáveis hidrológicas, conforme destacado por Burn \& Elnur (2002). 
Tabela 3. Valores de $\mathrm{K}, \mathrm{h}_{(\mathrm{dia}, 2)}$ e $\mathrm{h}_{(60,2)}$ encontrados para cada estação meteorológica, valores médios e coeficiente de variação (CV - \%) em cada região

\begin{tabular}{|c|c|c|c|}
\hline Local & $\mathrm{h}_{(\mathrm{dia}, 2)}$ & $\mathrm{K}$ & $\mathrm{h}_{(60,2)}$ \\
\hline \multicolumn{4}{|l|}{ Região Norte } \\
\hline Teófilo Otoni & 71,3 & 0,64 & 45,6 \\
\hline Pirapora & 69,5 & 0,71 & 49,4 \\
\hline Salinas & 66,4 & 0,72 & 47,8 \\
\hline Januária & 74,6 & 0,57 & 42,5 \\
\hline Montes Claros & 69,4 & 0,65 & 45,1 \\
\hline Média & 70,2 & 0,66 & 46,1 \\
\hline CV $(\%)$ & 4,3 & 9,2 & 5,7 \\
\hline \multicolumn{4}{|l|}{ Região Sul } \\
\hline Lavras & 77,9 & 0,64 & 49,9 \\
\hline Machado & 62,2 & 0,65 & 40,4 \\
\hline Caxambu & 74,4 & 0,48 & 35,7 \\
\hline Barbacena & 58,0 & 0,63 & 36,5 \\
\hline Média & 68,1 & 0,60 & 40,6 \\
\hline $\mathrm{CV}(\%)$ & 14,0 & 13,4 & 16,0 \\
\hline \multicolumn{4}{|l|}{ Região do Triângulo Mineiro } \\
\hline Capinópolis & 81,7 & 0,53 & 43,3 \\
\hline Uberlândia & 75,5 & 0,71 & 53,6 \\
\hline Uberaba & 81,1 & 0,68 & 55,1 \\
\hline Araxá & 69,2 & 0,72 & 49,8 \\
\hline Patos de Minas & 77,2 & 0,61 & 47,1 \\
\hline Média & 76,9 & 0,65 & 49,8 \\
\hline $\mathrm{CV}(\%)$ & 6,6 & 12,3 & 9,6 \\
\hline \multicolumn{4}{|l|}{ Região Leste } \\
\hline Viçosa & 76,3 & 0,55 & 41,9 \\
\hline Caratinga & 66,3 & 0,62 & 41,1 \\
\hline Aimorés & 68,6 & 0,65 & 44,6 \\
\hline Governador Valadares & 84,4 & 0,67 & 56,6 \\
\hline Média & 73,9 & 0,62 & 46,1 \\
\hline $\mathrm{CV}(\%)$ & 11,1 & 8,5 & 15,6 \\
\hline \multicolumn{4}{|l|}{ Região Centro } \\
\hline Bambuí & 79,3 & 0,61 & 48,4 \\
\hline Sete Lagoas & 68,5 & 0,62 & 42,5 \\
\hline Belo Horizonte & 89,4 & 0,49 & 43,8 \\
\hline Diamantina & 84,5 & 0,49 & 41,4 \\
\hline Média & 80,4 & 0,55 & 44,0 \\
\hline $\mathrm{CV}(\%)$ & 11,1 & 13,1 & 7,0 \\
\hline
\end{tabular}

Avaliando-se, ainda, a variabilidade do parâmetro $\mathrm{h}_{(60,2)}$, verifica-se que os coeficientes de variação são baixos o suficiente para se sugerir as médias aplicadas como bons estimadores a serem adotados para este parâmetro, quando da aplicação dos modelos em localidades inseridas dentro de cada região. Contudo, Vieira et al. (1991) encontraram coeficientes de variação superiores a 30\% para o Estado de São Paulo, trabalhando com um número maior de estações, estudando chuvas máximas diárias e concluindo que no estudo desta variável hidrológica, é pertinente considerar-se a variabilidade espacial. Souza Filho \& Oliveira (1996) destacam ainda dois motivos: melhorar a qualidade das predições, principalmente dentro do raio do alcance, e economizar em termos de amostragem. Na Figura 2 observa-se que o estudo da dependência espacial do parâmetro proporcionou um semivariograma com bom ajuste do modelo esférico (Eq. 6) mostrando que a variabilidade espacial deve ser considerada na determinação da chuva de projeto.

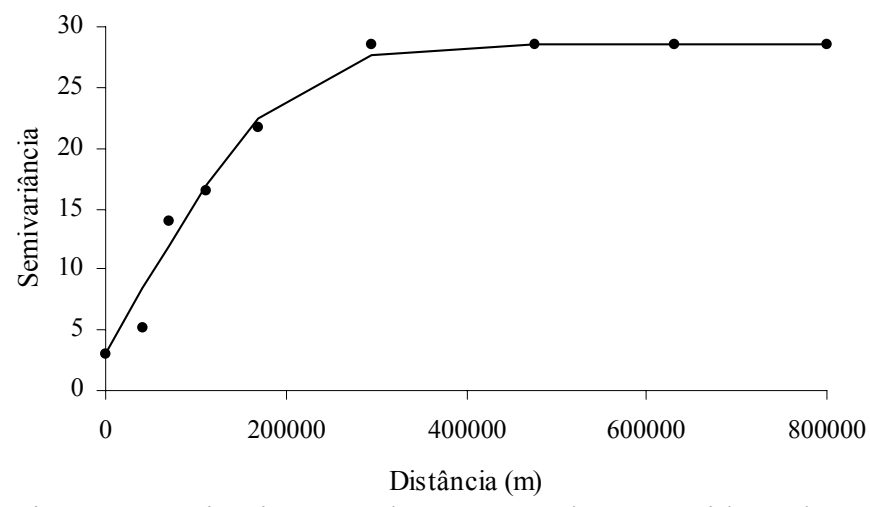

Figura 2. Semivariograma do parâmetro $\mathrm{h}_{(60,2)}$ considerando-se todas as estações meteorológicas utilizadas neste trabalho

$\gamma(\mathrm{h})=3+25,52\left[1,5\left(\frac{\mathrm{h}}{293685}\right)-0,5\left(\frac{\mathrm{h}}{293685}\right)^{3}\right]$

sendo que:

$\gamma(\mathrm{h})$ - representa a semivariância e h a distância euclidiana em metros

$\mathrm{Na}$ Tabela 4 tem-se as três estações da região Norte utilizadas para validação, com as distâncias em quilômetros, das estações utilizadas no ajuste do modelo para esta região, bem como o valor médio ponderado de $\mathrm{h}_{(60,2)}$.

Tabela 4. Estações meteorológicas da região Norte, respectivas distâncias euclidianas $(\mathrm{km})$ e valor médio ponderado de $\mathrm{h}_{(60,2}$

\begin{tabular}{lccc}
\multicolumn{1}{c}{ Estação } & Formoso & Arinos & Pedra Azul \\
\hline Teófilo Otoni & 624 & 536 & 202 \\
Pirapora & 295 & 181 & 449 \\
Salinas & 465 & 416 & 120 \\
Januária & 219 & 186 & 356 \\
Montes Claros & 328 & 252 & 295 \\
\hline $\mathrm{h}_{(60,2)}$ médio & 45,3 & 45,9 & 46,8 \\
\hline
\end{tabular}

Na Tabela 5 encontram-se os valores dos pesos de krigagem e o valor de $\mathrm{h}_{(60,2)}$ preditos pela geoestatística. Observa-se que em cada localidade utilizada para validação, há um número diferente de estações, devido ao alcance encontrado (294 km) haja vista que a dependência espacial somente é verificada a distâncias inferiores a este valor.

Tabela 5. Cidades utilizadas na vizinhança de krigagem, respectivos pesos de krigagem e valores de $\mathrm{h}_{(60,2)}$

\begin{tabular}{lccc}
\hline \multicolumn{1}{c}{ Estação } & Formoso & Arinos & Pedra Azul \\
\hline Pirapora & 0,558 & 0,337 & -- \\
Januária & 0,442 & 0,354 & -- \\
Montes Claros & -- & 0,309 & 0,503 \\
Teófilo Otoni & -- & -- & 0,497 \\
\hline $\mathrm{h}_{(60,2)}$ & 46,3 & 45,6 & 45,3 \\
\hline
\end{tabular}

A Figura 3 e a Tabela 6 permitem analisar-se o comportamento dos seus erros e os valores médios proporcionados pelas metodologias adotadas para cálculo do parâmetro $\mathrm{h}_{(60,2)}$. 


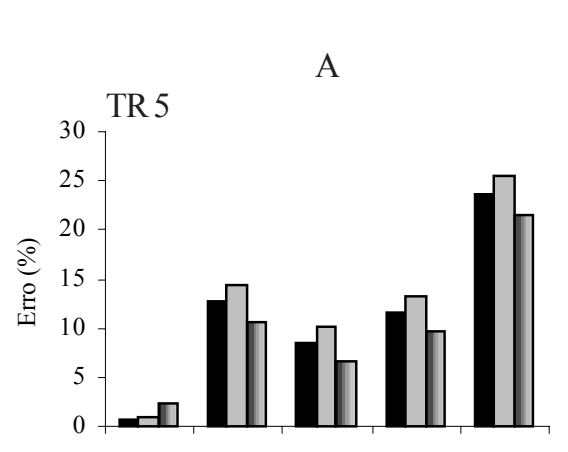

$\underset{\mathrm{B}}{\operatorname{Erro}}{ }^{*} \underset{\mathrm{Erro}}{* *} \square$ Erro $* * *$

$\mathrm{C}$
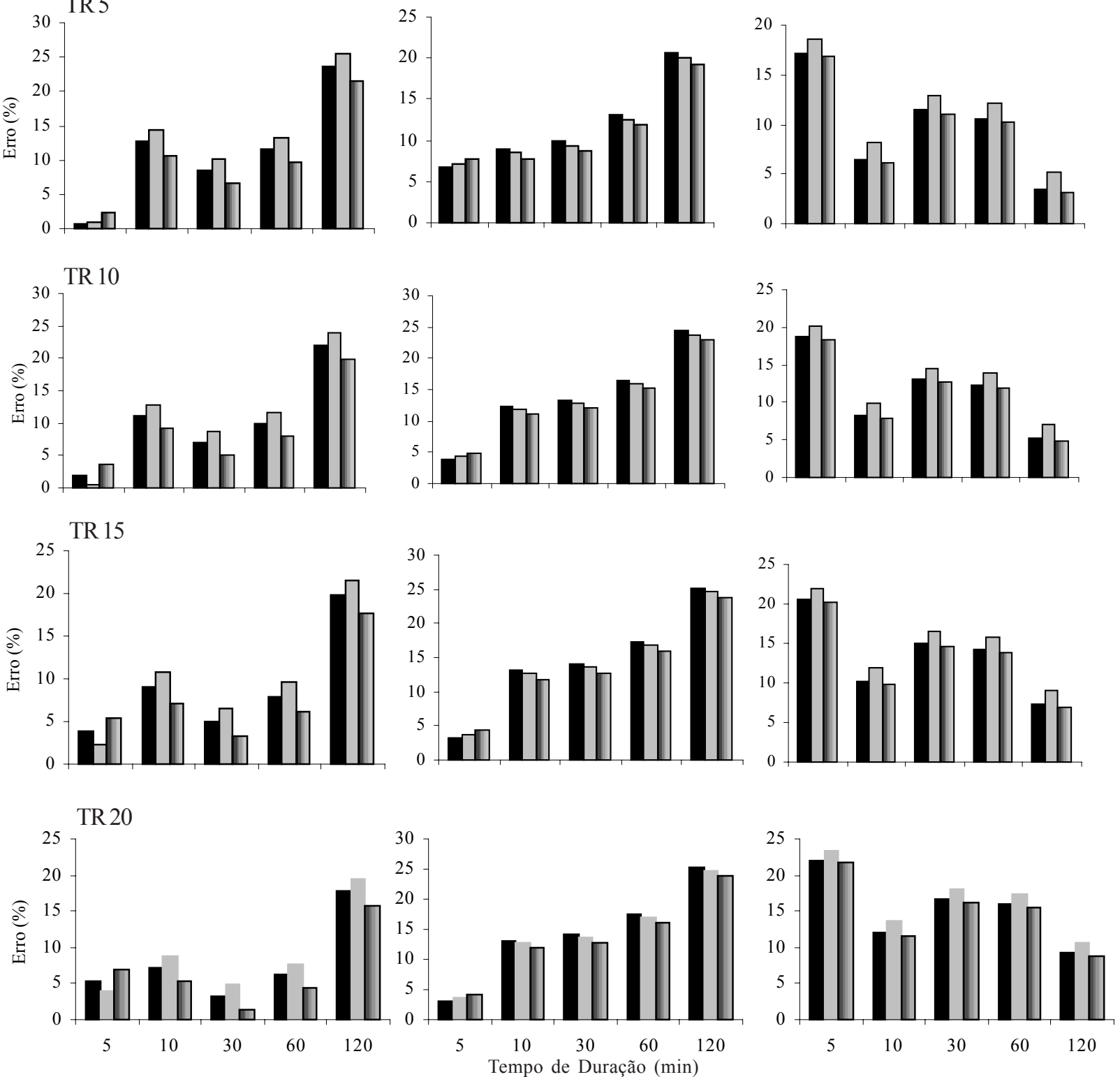

Erro * - erro médio gerado pelo modelo, considerando-se média aritmética na obtenção de $\mathrm{h}_{(60.2)}$; Erro ** - erro médio gerado pelo modelo, considerando-se média ponderada pelo inverso do quadrado da distância na obtenção de $\mathrm{h}_{(60.2)}$; Erro *** - erro médio gerado pelo modelo, considerando-se as prediç̧ões feitas pela krigagem na obtenção de $\mathrm{h}_{(60.2)}$

Figura 3. Comportamento dos erros produzidos pelas metodologias de avaliação de $\mathrm{h}_{(60,2)}$ para as estações de Pedra Azul (A), Arinos (B) e Formoso (C) para diferentes tempos de retorno $\left(\mathrm{TR}_{\text {anos }}\right)$

Tabela 6. Erros médios gerados pelo modelo da região Norte, em percentagem, considerando-se os métodos descritos para obtenção do parâmetro $\mathrm{h}_{(60,2)}$

\begin{tabular}{lccc}
\hline Estação & Erro * & Erro ** & Erro *** \\
\hline Formoso & 12,1 & 13,7 & 11,7 \\
Arinos & 11,6 & 11,3 & 10,9 \\
Pedra Azul & 9,7 & 10,9 & 8,5 \\
\hline
\end{tabular}

Analisa-se que a krigagem proporcionou os menores erros médios, o que pode ser também observado na Tabela 6 , na qual constam esses valores; no entanto, as figuras mostram alguns comportamentos peculiares a cada uma das estações.

Para a estação Pedra Azul (Figura 3A) a krigagem ensejou os menores erros para todos os tempos de duração, exceção feita ao tempo de $5 \mathrm{~min}$, cujo comportamento foi comum a todos os tempos de retorno. Os maiores erros foram obtidos para o tempo de duração de $120 \mathrm{~min}$, os quais ficaram em torno de 15 a $25 \%$, independentemente do tempo de retorno e do método empregado, porém a krigagem apresentou erros inferiores aos demais métodos.

Avaliando-se o comportamento dos erros para a estação de Arinos (Figura 3B) a krigagem proporcionou os menores erros, com exceção do tempo de duração de $5 \mathrm{~min}$, de maneira semelhante ao comportamento de Pedra Azul. Também, de forma semelhante, obtiveram-se os maiores erros para o tempo de duração de 120 min observando-se, no entanto, melhor desempenho da média aritmética sobre a média ponderada pelo inverso do quadrado da distância, para o tempo de duração de 
$5 \mathrm{~min}$. As diferenças entre os erros médios obtidos foram as menores para essa estação (Tabela 6) o que também pode ser observado na Figura 3B.

Para a estação de Formoso (Figura 3C), o comportamento dos erros foi diferente das estações anteriores, constatandose primeiramente que a krigagem ofereceu os menores valores para todos os tempos de duração e tempos de retorno; no entanto, os maiores erros foram para o tempo de duração de $5 \mathrm{~min}$, que ficou em torno de $20 \%$. Da mesma forma anterior, a krigagem gerou os menores erros, seguida pela média aritmética e pelo inverso do quadrado da distância.

Com base na avaliação da Figura 3 e Tabela 6, concluir-se que o método de Bell pode ser aplicado, já que os erros proporcionados em relação aos valores originais foram baixos, em especial para tempos de duração e tempos de retorno mais freqüentemente aplicados a projetos. Além disso, comprovouse que a krigagem é o método estatístico de predição que gera os melhores comportamentos no tocante a erros, em que os mesmos foram inferiores em todas as situações observadas, com poucas exceções. Deve-se considerar que a metodologia foi testada apenas para a região Norte, devido ao pequeno número de estações disponível (Tabela 1) para as demais regiões ou, quando disponíveis, as equações de chuvas intensas eram baseadas em séries históricas antigas, anterior a 1985, não oferecendo, portanto, nenhuma confiabilidade em termos comparativos. Mesmo assim, é razoável considerar-se, com base nos parâmetros e análises das qualidades matemáticas dos modelos, que se pode obter comportamento semelhante ao das estações testadas, quando se aplicar esta metodologia a outras regiões.

\section{CONCLUSÕES}

1. Os modelos ajustados são eficientes para predição da chuva de projeto, desde que sejam obedecidos os limites para tempo de retorno e tempo de duração.

2. O uso da krigagem geoestatística pode produzir resultados mais precisos e deve ser considerado na estimativa da chuva intensa padrão $\left(\mathrm{h}_{(60,2)}\right)$.

\section{LITERATURA CITADA}

Almeida, R.M.B. Características climatológicas do regime de chuvas em Minas Gerais. Viçosa: UFV, 1995. 64p. Dissertação Mestrado

Assad, M.L.L.; Assad, E.D.; Evangelhista, B.A. Chuvas extremas na região dos cerrados. In: Assad, C.D. (coord.) Chuva no cerrado: análise e espacialização. Brasília: EMBRAPA/ CPAC, 1994. p.49-54.

Bell, F.C. Generalized rainfall-duration-frequency relationships. Journal of the Hydraulics Division, New York, v. 95, n.1, p. 311-327, 1969.

Bertoni, J.C.; Tucci, E.M. Precipitação. In: Tucci, C.E.M., (org.). Hidrologia: ciência e aplicação. Porto Alegre: EDUSP/ABRH, 2001. Cap.5, p.177-241.

Brooks, K.N.; Ffolviott, P.F.; Gregersen, H.M.; DeBano, L.F. Hydrology and the management of watersheds. 2.ed. Ames: Iowa State University Press, 1997. 502p.
Burn, D.H.; Elnur, M.A.H. Detection of hydrologic trends and variability. Journal of Hydrology, Amsterdam, v.255, n.1-4, p.107-122, 2002.

Chow, V.T.; Maidment, D.R.; Mays, L.W. Appied hydrology. New York: McGraw-Hill, 1988. 572p.

Cruciani, D.E.; Machado, R.E.; Sentelhas, P.C. Modelos da distribuição temporal de chuvas intensas em Piracicaba, SP. Revista Brasileira de Engenharia Agrícola e Ambiental, Campina Grande, v.6, n.1, p.76-82, 2002.

Denardin, J.E.; Freitas, P.L. Características fundamentais da chuva no Brasil. Pesquisa Agropecuária Brasileira, Brasília, v.17, n.10, p.1409-1416, 1982.

Froehlich, D.C. Short-duration-rainfall intensity equations for drainage design. Journal of Irrigation and Drainage Engineering, Reston, v. 119, n.5, p. 814-828, 1993.

Gallant, A.R. Non linear statistical models. New York: J. Wiley, 1987.610p.

Gotway, C.A.; Ferguson, R.B.; Hergert, G.W.; Peterson, T.A. Comparison of kriging and inverse-distance methods for mapping soil parameters. Soil Science Society of America Journal, Madison, v. 60, n.4, p.1237-1247, 1996.

Haan, C.T. Statistical methods in hidrology. 2. ed. Ames: Iowa State University Press, 1979. 377p.

Heneker, T.M.; Lambert, M.F.; Kuczera, G. A point rainfall model for risk-based design. Journal of Hydrology, Amsterdan, v.247, n.1, p. 54-71, 2001.

Mello, C.R.; Ferreira, D.F.; Silva, A.M.; Lima, J.M. Análise de modelos matemáticos aplicados ao estudo de chuvas intensas. Revista Brasileira de Ciência do Solo, Viçosa, v.25, n.3, p.693-698, 2001.

Moreira, I.P.S. Classificação ecológica do território brasileiro situado entre 16 e $24^{\circ}$ latitude sul e 48 e $39^{\circ} 51^{\prime}$ longitude oeste. Viçosa: UFV, 1997. 156p. Tese Doutorado

Nobukuni, P. Análise das precipitações com duração de 5 minutos a 24 horas, nas regiões de Jaboticabal e Presidente Prudente. Jaboticabal: UNESP/FCAV, 2000. 65p. Dissertação Mestrado

Pegoraro, R.C.F. Análise de equações de chuvas intensas generalizadas. Campinas: UNICAMP, 1996. 110p. Dissertação Mestrado

Pinto, F.A. Chuvas intensas no estado de Minas Gerais: análises e modelos. Viçosa: UFV, 1995. 87p. Tese Doutorado

Pinto, F.A.; Ferreira, P.A.; Euclydes, H.P. Chuvas intensas e altura de chuva para projeto de drenagem do solo para a Bacia do Rio Doce, Minas Gerais. In: Ruralminas/UFV. Parte 2, Viçosa, 1996, p.99-137.

Righetto, A.M. Hidrologia e recursos hídricos. São Carlos: EESC/USP, 1998. 840p

SAS - Statistical Analyses System. Language guide for personal computers. 6. ed. Cary: SAS Instituto, 1985. 429p.

Silva, D.D.; Valverde, A.E.L; Pruski, F.F.; Gonçalves, R.A.B. Estimativa e espacialização dos parâmetros da equação de intensidade-duração-freqüência da precipitação para o Estado de São Paulo. Revista Engenharia na Agricultura, Viçosa, v.7, n.2, p.70-87, 1999. 
Souza Filho, M.D.I. de; Oliveira, M.S. de. Planejamento da amostragem de solos visando a estimação da média espacial do teor de cálcio utilizando a geoestatística. Ciência e Agrotecnologia, Lavras, v. 20, n.3, p.387-393, 1996.

Thompson, S.K. Sampling. 1.ed. New York: A Wiley-Interscience Publication, 1992.343p.

Vieira, S.R. Geoestatística em estudos de variabilidade espacial do solo. In: Novais, R. F.; Alvarez V, V.H.; Schaefer, C.E.G.R. (eds). Tópicos em ciência do solo. Viçosa: Sociedade Brasileira de Ciência do Solo, 2000. cap.1, p.1-54.
Vieira, S.R.; Lombardi Neto, F.; Burrows, I.T. Mapeamento da chuva diária máxima provável para o estado de São Paulo. Revista Brasileira de Ciência do Solo, Campinas, v.15, n.1, p.93-98, 1991.

Yevjevich, V. Probability and statistics in hydrology. Littleton: Water Resources Publications, 1982.302p.

Yue, S. The Gumbel logistic model for representing a multivariate storm event. Advances in Water Resources, Amsterdam, v.24, p. 179-185, 2001 